\title{
Experimental Phonetics in Applied Linguistic Research
}

\author{
Denis Martyanov ${ }^{1 *}$, Ravza Kulsharipova ${ }^{2}$, Nadezhda Oglezneva ${ }^{3}$ \\ 1,2,3 Kazan Federal University \\ *Email: damartyan@yandex.ru
}

\section{Received: 15th December 2017, Accepted: 20th December 2017, Published: 31st December 2017}

\begin{abstract}
The formation of experimental phonetics was accompanied by an interest to the physical nature of speech sounds. The experimental methodology makes it possible to detail the characteristics of sound formation mechanisms. The foundations of experimental phonetics were first laid at Kazan University at the end of the 19th century. Even then, the phonetic structures of the language were identified as paramount, due to which it became possible to substantiate, creatively and methodologically correctly, the theory and methodology of experimental study of the sound structure of the language, and to predict milestones and steps of future research. Extralinguistic views were largely transmitted through phonetic structures and units. It is really important to assess and reveal the role of phonetics in the formation of the scientific knowledge system, because in the conditions of multilingualism, the sounds of native speech implicitly act as a means to formalize mental spaces (according to the tradition of Kazan Linguistic School, this can be a model of the word phonemography). Acoustic phonetics helps to reveal the general patterns of the dynamics of the speech model in communication between the speakers of heterogeneous languages and to identify vulnerable points in the articulatory base, using the acoustic parameters of sounding speech elements. Modern instrumental research is aimed at describing the acoustic component of speech, which contributes to a further in-depth study of human speech phenomenon as a complex associative aggregate (the definition of V.A.Bogoroditsky, the founder of experimental phonetics in Russia). Our research is aimed at revealing the role and potential of experimental phonetics in the process of phonetic knowledge formation.
\end{abstract}

Keywords: Experimental Phonetics, Acoustic Segmentation, Phonemography of the Word, Basic Acoustic Correlates, Speech Signal, Phonetic Portrait of the Speaker

\section{Introduction}

Philological competence includes a database on all systemic knowledge for a philologist. The theory of knowledge is widely studied in many scientific disciplines, in particular in psycholinguistics, cognitive linguistics, linguistic culturology, etc. Y.I.N. Boduen de Courtenay, the founder of Kazan Linguistic School, created one of the first classifications devoted to the types of knowledge in linguistics. He recommended to distinguish such types of knowledge as intuitive, scientific, linguistic in the process of studying the language.

Later, in connection with the expansion of the approaches to the fundamental paradigms of the language, for example the system-structural, communicative, with the development of the activity aspect, the typology of knowledge acquired a detailed character in the following parameters: relation to science; social life; research methodology; dynamics of verbal representation, etc.; these aspects are described in details in the works of V.V. Krasnykh [Krasnykh 2012: 172-175].

A systematic approach to the study of the phenomena and structures of language was also originally set in the concept of Kazan Linguistic School. We believe that this was the reason for the interest in understanding the phonetic level of language as a harmonic part of linguistic unity. Resources, the possibilities of the phonetic system of the language were interpreted within the framework of psychophonetics, anthropophonics, phonemography of the word.

These approaches did not simply reflect the metalinguistic entities - they were a kind of indication of the directions to studying phonetic units within the framework of science and the everyday communication. At the same time, these aspects constituted a platform for applied analysis of phonetics. It was at Kazan University at the end of the 19th century that it became possible to substantiate the theory and methodology of experimental study of the sound structure of the language, to predict the milestones and steps of future research. V.A. Bogoroditsky outlined the key moments of instrumental research which play a leading role in developing the foundations of the articulatory base of language and speech [Bogoroditsky 1930].

In the linguodidactic aspect, linguistic knowledge is not only the criteria for mastering the language, it structures the integral picture of mental spaces of the native speaker and thereby significantly diversify the vectors of the human speech continuum. We are of the opinion that the basis and dynamics of human cognitive skills should be interconnected with the laws of speech, so relevant for our days.

V.A.Bogoroditsky defined speech as a complex associative aggregate. The phonetic component of speech predetermines a lot in the formation of both 
the corresponding metadata and the basic means of organizing and transmitting acoustic-phonetic and pragmatic information. The growing interest to speech implies the development of such a competence in a philologist that would allow him/her to successfully implement a wide range of problems that originate in the sound structure of the language and through it emerge to create communicative tactics and strategies: models of communication, phonetics and presuppositions; phonetics and universal-object code, etc. [Dediu 2017].

\section{Materials and Methods}

Experimental phonetics at the initial stage of its development started with the study of neurophysiological mechanisms. In Kazan linguistic school in accordance with the results of studies of that time there were obtained the results important for our time: speech zones in the cortex of the human brain; the nature of linguistic thinking; the causes of individual speech phenomena such as slips of the tongue etc.

At the theoretical level, it must be explained what causes the appearance of these phenomena. According to the representatives of Kazan linguistic school, experimental recordings contribute to improving the skills not only of motor perception of speech, but also to association, especially as the essence of associations by similarity and contiguity played a big role in developing a program of experimental phonetic description of the language by the representatives of Kazan linguistic school. We also consider these approaches to be meaningful and expedient in the process of teaching the basic mechanisms of the phonetic structure of the language.

The basic types of speech activity of a person are speaking, listening, writing, reading. Representatives of Kazan linguistic school interpreted sound and writing as two vital ways of language. In the conditions of polylingualism, the speakers sometimes ignore the actions of these modes which reflect the dynamics of human speech, its ability to extract basic and additional meanings from phonetic and acoustic information.

The visual series of permissible slips of the tongue and hooks obtained by means of computer programs Speech Analyzer or PRAAT allows students to specify the phase features of variable sounds, the oscillograms help to understand the phonetic level in the causes of these phenomena. Orthophonic variability is also important for setting the articulation base for those who learn a foreign language as a non-native or foreign. With orthophony, the possibility of modeling non-tonal accents (the terms of S.V.Kodzasov), which also reveals the emotional potential of speech, correlates with the orthophony, the intensity curves in this case really indicate how well the speaker perceives the emotional, axiological parameter of speech.

\section{Results}

The rhythmic organization of speech is indicative for explanation of the semantic projection of an utterance - this is provided by using the so-called integrative description. Automatic programs in describing the speech signal are necessary for the training of rhythmic, dynamic and melodic speech characteristics. Computer technologies allow the researchers to make a phonetic portrait of a native speaker according to the social rank, to clarify the dynamics of speech changes with the involvement of ethnic and cultural factors. In the process of learning, we can trace what trends occur in the development of the articulatory base. The degree of normality of these parameters is rather conditional, but they are extremely important when stating a phonemic representation in the conditions of polylingualism, in particular, such a language situation is currently observed in the Republic of Tatarstan; we consider it advisable to pay attention to the variation of speech signal parameters depending on the basic extralinguistic factors (age, emotional state, education, culture, etc.) when performing a comparative analysis of acoustic data. The received acoustic data will be important also at the decision of adaptation questions in dialogue between different native speakers. This project will contribute to the preservation and development of languages in conditions of multilingualism.

In parallel with the oscillographic observations of the sound structure of the word, modern experimental phonetics solves the problems of the mechanisms of listening to speech through a package of prosodic speech characteristics. It becomes possible to reproduce the text with the given parameters; by the degree of naturalness of utterance, accent, genre, psychology, etc. The acoustic data obtained with the help of these computer programs make it possible to carry out a comparative analysis of the phonetic structure of the language and native language, while observing both phonetic universal and unique features. The peculiarities of the accent revealed in this case will also indicate the state of phonological hearing of speaking, stable skills to perceive and understand speech using sound and acoustic registers.

Comparative phonetic bases of different languages are aimed to solve not only the didactic, methodological problems themselves, but also to replenish the phonetic fund of the languages in a certain region. Nowadays the creation of such phonetic funds in the Republic of Tatarstan is in the process; in the 90s of the XX century, the phonetic 
fund of the Russian language was created by the joint efforts of scientists from Moscow, St. Petersburg and Germany.

The phonetic fund of the Republic of Tatarstan is of interest not only in linguodidactical perspective, but also in applied aspect (clinical linguistics, neurolinguistics) [Richards 2015; Gorobets 2015, 2016], forensic linguistics [Lacerda 2013]).

\section{Discussion}

Experimental phonetics is able to correct the dynamics of the phonetic space of speech in native speakers depending on their social parameters; the aspects of social phonetics are refined due to experimental technologies, mainly such characteristics as melodic, prosodic timbre, rhythmdynamic modeling in speech behavior of speakers, actualization of meaning with the help of prosodic markers, which is recognized by all specialists in applied phonetics [Kodzasov 2009; Potapov, Potapov 2012].

In the process of teaching the basics of phonetics, the production of suprasegmental phonetics requires the involvement of a large number of techniques; computer programs allow real enlargement of semantic functions of speech prosody. Theoretically, we consider the ideas of L.V.Zlatoustova [Zlatoustova 1981], S.V.Kodzasov [Kodzasov 2009], R.K.Potapova, V.Potapov [Potapova, Potapov 2012] and others.

Phonetic transcription operates already established principles, the prosodic intonation is still variable. If we compare the algorithms of prosodic transcription according to S.V. Kodzasov and the works of St. Petersburg phonetic school, certain variations will be revealed, and the experimental analysis will help to refine a number of methods, for example, a set of phrase accents or accent types [Sajin 2017]. Accents will be brighter presented in the practice of learning by visualizing the intonational contours of speech; intonograms are very indicative in this regard. It will be more difficult to mark the phonation; here it is necessary to emphasize the tasks of training the prosodic timbre with modeling the pitch of the voice and the dynamic characteristics of the sounds. The pitch range over the sound in the speech stream will be indicative in the development of the skills to divide speech stream into segments. This is especially important when it comes to the formation of a system of phonetic knowledge among nonnative speakers. We attempted to apply the data of experimental phonetics to teaching non-Russian speaking students the basics of Russian phonetics, taking into account the traditions of Kazan linguistic school, which gave good results in the field of syntagmatic formalization of speech, localization of phrasal stresses, correlation of sound and meaning.
The registration of the range of tonal configurations with the help of experimental studies in speech is not only actual in educational and methodical aspects, but also solves a number of applied questions, enriching the philological competence of students; formalization of speech through acoustic sound supports, their perception and interpretation with a semantic program that reveals the general semioticcognitive potential of speakers. In most works on segmentation of speech, special attention is paid to the problems of syllabic prosody. The acoustic parameters of the syllable are the frequency of the pitch on the border areas, the intensity curve, the duration - all this also contributes to the development of listening skills, correction and coordination of sounding / pronunciation and understanding techniques, thereby achieving success in developing skills and skills in working with phonetic material of varying degrees in semantic and communicative complexity.

The recognition of sounding speech markers is a part of the general phonetics of the language, when through the mechanisms of external speech a person comprehends the meaning of the utterance and identifies the semantic focus of lexemes; experimental phonetics contributes to the elaboration of dividing the semantic parts in speech by means of physical parameters. Thus, the formant characteristics allow researchers not only to determine the purity of the background gesture in sound formation, but also to point out certain paralinguistic nuances, since the shift of the spectrum into the high or low frequency region indicates both the sound quality and the presence of the extralinguistic plan. The emotional component of experimental phonetics also helps to solve the tasks of identifying a person by voice. The typology of prosodic models in Russian phonetics was successfully studied by such scientists as L.V.Zlatoustova, R.F.Kasatkina, S.V.Kodzasov, R.K.Potapova, N.D.Svetozarova, and others. It is important to use the resources of experimental phonetics in the conditions of polylinguism [Fenwick 2017] - this situation takes place in the Republic of Tatarstan, when two different languages actively interact in a single communicative space, which have been contacting each other for several centuries, as a result of which the so-called "mixed character of the language" (in terminology Y.I.N. Boduen de Courtenay) was formed.

The details of phonetic acoustic information can be obtained through the use of experimental phonetics technologies (currently it is available through computer programs PRAAT, and Speech Analyzer). The programs provide acoustic characteristics of the speech signal in one complex; on the screen you can 
simultaneously see oscillograms, intensity curves, melodies, spectral contours.

\section{Conclusions}

The data of experimental phonetic analysis of speech is of great importance in teaching the basics of speech activity. Acoustic phonetics helps not only to reveal the general patterns of the dynamics of the speech model in communication between native speakers of heterogeneous languages, but also to identify vulnerable points in the articulation base using the acoustic parameters of sounding speech elements, to make up a kind of phonetic code for extracting information from the flow of speech.

Thus, experiments on the perception of the semantic segments of speech showed a small percentage of accentuation in the sounding speech of non-speakers of the Russian language; if you include the emotional factor, then there is a better sensitivity to the semantic points of the message, so in teaching practice, this technique should be actively used with visualization of data on the screen. Semantics of accentuation in Russian linguistics was developed by T.M.Nikolaeva [Nikolaeva 2000].

The auditive analysis also revealed the advisability of applying exercises for modeling frequency ranges of the fundamental tone in the practice of teaching and the formation of phonetic knowledge. This is especially important when working with native speakers of tonal languages, for example, Chinese. But for Tatar native speakers this is also significant, because rhythmically Russian and Tatar languages are different; they can be qualified as accent and anaccent, accordingly, the rhythm-melodic organization will differ. The typology of accent patterns will have different rhythmic, dynamic and melodic structures. Experimental phonetics appears in such a process not as an auxiliary didactic device, but as an integral, semantic and grammatical information technology that combines the semantic and communicative competencies of learners.

Our research is carried out according to modern principles of interpretation of acoustic phonetics possibilities. V.A. Bogoroditsky's theory (speech is a complex associative phenomenon) is confirmed in modern linguistics by the developed modern computer technologies which should be used in the educational process.

\section{Acknowledgements}

The work is performed according to the Russian Government Program of Competitive Growth of Kazan Federal University.

\section{References}

Bogoroditsky V.A. Phonetics of the Russian language through a prism of experimental data.
Kazan: Publishing House of the Tatar Culture, 1930. $357 \mathrm{p}$.

Zlatoustova L.V. Phonetic units of Russian speech. Moscow: MSU, 1981. 105 pp.

Potapova R.K., Potapov V.V. Speech communication: From sound to utterance. M.: Languages of Slavic Cultures, 2012. 464 p.

Kodzasov S.V. Research in the field of Russian prosody. M.: Languages of Slavic Cultures, 2009. $496 \mathrm{p}$.

Krasnykh V.V. Fundamentals of psycholinguistics. Moscow: Gnosis, 2012. 333 p.

Nikolaeva T.M. From sound to text. Moscow: Languages of Russian culture, 2000. 680 p.

Dediu D., Janssen R., Moisik S.R. Language is not isolated from its wider environment: Vocal tract influences on the evolution of speech and language // Language and Communication. 2017. Vol. 54. Pp. 9-20.

Fenwick S.E., Best S.T., Davis C., Tyler M.D. The influence of auditory-visual speech and clear speech on cross-language perceptual assimilation // Speech Communication. 2017. Vol. 92. Pp. 114-124.

Gorobets, E.A., Kulsharipova, R.E., Lotfullina, N.Z. Semantico-phonological disorders in patients with Wernicke's aphasia // Social Sciences (Pakistan). 2015. 10 (5), pp. 566-570.

Gorobets, E., Kulsharipova, R., Novak, M. Speech disorders in patients with cognitive impairment caused by neurogenerative diseases: An overview // Journal of Language and Literature. 2016.7 (2), pp. 177-180.

Lacerda F. Voice stress analyses: Science and pseudoscience // Proceedings of Meetings on Acoustics. 2013. №19.

Richards S., Goswami U. Auditory processing in specific language impairment (SLI): Relations with the perception of lexical and phrasal stress // Journal of Speech, Language, and Hearing Research. 2015. 58(4), c. $1292-1305$

Sajin S.M., Connine C.M. The influence of speech rate and accent on access and use of semantic information // Quarterly Journal of Experimental Psychology. 2017. 70(4). Pp. 619-636. 